Background Status epilepticus is a neurological emergency and is refractory to standard treatment at times. New antiepileptic drugs have been introduced but the place of application of these drugs in status is yet to be established.

Objective To compare efficacy and adverse effects of intravenous Valproate and intravenous Levetiracetam as second line anti-epileptic drugs in status epilepticus to intravenous Phenytoin.

Methodology 42 patients between 6 months to 12 years presenting with status epilepticus were included in the study and randomly distributed in three groups depending on the day of admission and each group was assigned one of three drugs under study as second line anti-epileptic drug. The ability of the drug to stop convulsions as well as time taken to stop convulsions and adverse effects were noted. Analysis of the data was done using chi square test.

Results $57 \%$ patients studied for phenytoin(12/21), $63 \%$ for valproate $(7 / 11)$ and $80 \%$ for levitracetam $(8 / 10)$ became non convulsive after the use of respective drugs. But on applying chi-square test, $p$ value showed that the comparison was not significant. The average time taken to stop convulsions by phenytoin is $10 \mathrm{~min}$, valproate is $10 \mathrm{~min}$ and by levetiracetam is $11 \mathrm{~min}$, which again was not statistically significant. Only 2 out of $42(4.7 \%)$ developed minor adverse effects from phenytoin in the form of excessive drowsiness and irritability.

Conclusion Clinically the efficacy of i.v. levetiracetam and i.v. valproate was found to be better than i.v. phenytoin but no statistically significant difference was observed. So phenytoin remains the preferred second-line anti-convulsant in status epilepticus with minimal side-effects.

\section{G53 CURRENT PRACTISE RELATING TO FLUID BOLUS ADMINISTRATION IN CRITICALLY ILL CHILDREN}

doi:10.1136/archdischild-2013-304107.065

G Sambandamoorthy, D Inwald, M Peters, P Ramnarayan. Children's Acute Transport Service, Great Ormond Street Hospital, London, UK

Aim To establish the frequency, amount and the diagnostic reasons for fluid bolus administration in children referred to a paediatric intensive care (PIC) retrieval service

Methods Retrospective case notes review of all referrals to a PIC retrieval service between May and October 2012 (6 months). Data collected included demographic details, reason for PIC referral, and total amount of bolus fluids given at referral. Blood products were excluded from the total fluid bolus calculations. In children retrieved by the service, additional data on total amount of fluid boluses given during retrieval and patient acuity (need for invasive ventilation, vasoactive agent use and PIM-2 score) were analysed. Categorical data are reported as counts (percentages) and continuous data as mean (standard deviation) or median (inter-quartile range) as appropriate.

Results During the 6-month study period, 1031 referrals were made to the retrieval service. At referral, 180 patients had received $\geq 20 \mathrm{mls} / \mathrm{kg}$ of fluid boluses $(17.5 \%$ ) and 54 had received $\geq 40 \mathrm{mls} / \mathrm{kg}$ $(5.2 \%)$. The main diagnoses for which children were given fluid boluses $\geq 20 \mathrm{mls} / \mathrm{kg}$ were sepsis $(30 \%)$ and status epilepticus $(21 \%)$. 548 children (53\%) were retrieved to PICU. Patients receiving $\geq 40 \mathrm{mls} / \mathrm{kg}$ by PICU admission had a median age of 14 months (IOR $2-47)$, were mostly male (60\%), requiring invasive ventilation $(98.3 \%)$ and vasoactive agents (61.5\%). The median PIM-2 predicted risk of mortality was $10 \%$ (IOR 6-19\%). A summary of total fluid administered at various time points is provided below in cases where a PIC retrieval team was mobilised $(n=548)$ :

\section{Abstract G53 Table 1}

\begin{tabular}{|c|c|c|}
\hline Timepoint & $\geq 20 \mathrm{mls} / \mathrm{kg}$ & $\geq 40 \mathrm{mls} / \mathrm{kg}$ \\
\hline At referral & $141(26 \%)$ & $47(9 \%)$ \\
\hline At retrieval team arrival at local hospital & $192(35 \%)$ & $83(15 \%)$ \\
\hline At the end of retrieval & $214(39 \%)$ & $109(20 \%)$ \\
\hline
\end{tabular}

Conclusion Our audit provides valuable baseline data on the frequency, amount and timing of fluid bolus administration in critically ill children with a range of diagnoses. At a time when fluid therapy is a subject of considerable debate, this study provides useful information on current practise and may help in the design of future interventional trials in this area.

\section{G54 SHOULD HYPERNATREMIA BE TAKEN SERIOUSLY IN THE PAEDIATRIC CRITICAL CARE SETTING?}

doi:10.1136/archdischild-2013-304107.066

'D Athavale, 'M Bradbury, ${ }^{2} \mathrm{~S}$ Playfor, ${ }^{1} \mathrm{M}$ Shenoy. ${ }^{1}$ Department of Paediatric Nephrology, Royal Manchester Children's Hospital, Manchester, UK; ${ }^{2}$ Paediatric Intensive Care, Royal Manchester Children's Hospital, Manchester, UK

Aims Hypernatremia in the critical care setting is frequently observed and is shown to be associated with higher mortality in adults. We studied the prevalence and factors surrounding the presence of hypernatremia in a paediatric critical care unit.

Method From April 2007 to March 2009, the presence of hypernatremia defined as sodium $(\mathrm{Na})$ greater or equal to $150 \mathrm{mmol} / \mathrm{l}$ was studied. Demographic details of patients, including the primary intensive care unit (ICU) diagnosis were noted. The duration and peak of hypernatremia along with fluid and diuretic management was reviewed.

Results 130 episodes of hypernatremia (10\%) were detected in a total of 1301 ICU admissions over this period. Excluding 14 patients who had a head injury as cause for admission requiring active maintenance of higher sodium levels, there were 116 ICU patient episodes of hypernatremia in 104 patients (52 male, 52 female). Hypernatremia ranged from 150 - $182 \mathrm{mmol} / \mathrm{l}$ (median $155 \mathrm{mmol} / \mathrm{l}$, IOR $152-158 \mathrm{mmol} / \mathrm{l}$ ) with a duration range of 1-16 days (median 2 days, IOR 1-3 days) in the entire group. Management was variable from no active intervention to increasing fluids to administrating diuretics. ICU mortality was $4.6 \%$ (55 deaths) in the non-hypernatremic group compared to $19.0 \%$ (22 deaths) in the hypernatremic group of patients $(p<0.0001)$.

Conclusion Hypernatremia in the critical care setting is common and appears to be associated with increased mortality. Fluid management in the sick child can be challenging and the clinician needs to be alerted to initiate optimal fluid management in the hypernatremic child.

\section{G55(P) AUDIT ON OPTIMAL POSITIONING OF PERIPHERALLY INSERTED CENTRAL CATHETERS IN NEONATES}

doi:10.1136/archdischild-2013-304107.067

${ }^{1}$ A Saha, ${ }^{2} \mathrm{R}$ Fernandez. 'Department of Paediatrics, Maidstone and Tunbridge Wells Hospitals NHS Trust, Maidstone, UK; ${ }^{2}$ Trevor Mann Baby Unit, Brighton and Sussex University Hospitals NHS Trust, Brighton, UK

Aims Changes in arm position has been known to cause migration of peripherally inserted central catheter (PICC) tips in neonates, however this relationship is poorly understood. An optimal placement of central catheter tip in neonates is essential to minimise life threatening complications like cardiac tamponade.

This study aims to ascertain the relationship between arm position and central catheter tip displacement in neonates, with an aim to subsequently develop a clinical guideline on the optimal use of these lines by minimising catheter tip migration and its complications.

Methods After ethical approval was granted by the Education Department at the Deanery, an audit was undertaken at a tertiary neonatal unit to collect data on paired radiographs in order to establish the relationship between the angle of the arm at the shoulder and correlating it with the location of the catheter tip as seen on plain radiography. The angle of the arm with respect to the midline 
of the body was carefully measured, and the location of the tip of the PICC lines was identified using agreed bony reference points, and its distance from the heart was measured. Paired X-rays (of the same baby) were compared with careful documentation of the perceived changes PICC line tip positions with respect to different angles of arm position.

Results A total of 32 pairs of X-rays that met our criteria were reviewed. Arm movements were associated with catheter displacement. For catheters placed in the basilic vein, there was a mean displacement of $0.17 \mathrm{~mm} /$ degree $(-0.53$ to +1.4$)$ towards the heart on adduction and $0.1 \mathrm{~mm} /$ degree $(-0.46$ to +0.4$)$ away from the heart on abduction of the arm. Similarly, for the cephalic vein, there was a mean displacement of $0.34 \mathrm{~mm} /$ degree $(-0.53$ to +1.6$)$ towards the heart on adduction and $0.32 \mathrm{~mm} /$ degree $(-0.8$ to +0.43$)$ away from the heart on abduction of the arm.

Conclusion Although this study did not establish any correlation in magnitude or direction, a clinically significant degree of catheter tip migration was observed with changes in arm position for each paired radiograph reviewed. A further prospective study under direct ultrasound visualisation is envisaged to study this relationship further.

\section{G56(P) HOW TO CONSTRUCT HI-FIDELITY ONLINE MEDICAL DEVICE SIMULATORS}

doi:10.1136/archdischild-2013-304107.068

C Bosman. Department of Neonatology, Nottingham University Hospitals NHS Trust, Nottingham, UK

Objectives I set out to investigate how the workings of a complex medical device could be visually represented and documented on paper, and then be translated into computer code to produce an online model.

The device chosen was a SiPAP ${ }^{\circledR}$ Infant Flow Driver (Carefusion, Ca) for providing nasal continuous positive airway pressure (NCPAP) ventilation to premature newborn infants. This device is used worldwide, and implements a touch screen control panel to set alarms and change settings.

Methods Statechart theory was designed in the late 1980s to diagram flight systems. This system was easy to learn and facilitates the conceptualization and illustration of both simple and, with practise, complex processes.

The Model-View-Controller (MVC) design pattern is a software engineering framework that requires the separation of the user interface from the functionality of the system. Using this pattern, the device was mapped by producing not one, but two statecharts one for the user interface of the device, and the other for my perception of the inner workings.

Adobe Flash (Adobe, $\mathrm{Ca}$ ) is a computer programme that is commonly used to create interactive multimedia web sites. Using the MVC design pattern I used FLASH to build up the physical 'View' of the device, and then coded the 'View Controller' and 'Model', by using the two statecharts as a map.

Results I discovered that by using statecharts and the MVC design pattern, both the inner workings and the user interface of a complex medical device could be represented and documented, then coded into a highly realistic working online simulator. The next stage is to create and implement a statechart for both training and assessment and finally compare the effectiveness with traditional clinical learning in a trial.

Conclusions Hi-fidelity online simulators of complex medical devices can be produced much more easily by harnessing the power of statechart theory with the flexibility of the Model-View-Controller design pattern.

Additional info The online simulator can be viewed at www. sipap.net.

\section{G57(P) GENDER MORTALITY DIFFERENCES OF INFANTS ON PICU? AN ELABORATION ON ADDITIONAL ANALYSIS}

doi:10.1136/archdischild-2013-304107.069

${ }^{1} \mathrm{~K}$ Amer, ${ }^{2} \mathrm{~S}$ Nadel, ${ }^{3} \mathrm{R}$ Basu-Roy. 'General Surgery, Kings College Hospital, London, UK; ${ }^{2}$ Paediatric Intensive Care, St Mary's Hospital, London, UK; ${ }^{3}$ Paediatrics, The Royal Berkshire Hospital, Berkshire, UK

Background Developed countries have exhibited higher rates of PICU admission and case fatalities in male infants compared to females (Scott Watson et al, Am J Respir Crit Care Med, 2003). Contrastingly, in addition to the disease-specific protection vaccination offers, observational studies and randomised trials in developing countries have indicated there may also be non-specific effects to such vaccines, particularly in females (Aaby et al, PIDJ, 2007). Specifically, the diphtheria-tetanus-pertussis (DTP) vaccine has been associated with poor growth and increased morbidity in girls (Agergaard et al, Vaccine, 2011). Recent studies continue to highlight female infant mortality following early DTP vaccination within developing countries (Abay et al, Arch Dis Child, 2012).

Aims Expanding last years database to 5 years of PICU admissions, we hypothesise that non-specific vaccine effects would be demonstrated by differences in gender mortality and admission to PICU in infants with life-threatening infection.

Method Using the UK PICANET database from January 2006 to December 2010 we gathered a database of 38,157 infants $<12$ months of age. Stringent criteria excluded planned admissions, noninfectious aetiologies, and infants of unknown gender. Cohorts where then categorised into those $>6$ months and those $<6$ months, with the assumption that all those $>6$ months of age have received their primary course of DTP/IPV immunisation. From this, we established mortality percentages for females and males admitted due to infectious causes within their age-defined groups.

Results Total infant PICU admissions due to infectious causes were greater in both male cohorts compared to female cohorts $(\mathrm{M}<6$-months $=3,592, \mathrm{~F}<6$-months $=2,468 ; \mathrm{M}>6$-months $=1,020$, $\mathrm{F}>6$-months $=781$ ). However, female mortality due to infectious causes for admission was greater than male mortality in both the under 6-month cohort $(\mathrm{F}=4.94 \%, \mathrm{M}=3.54 \%)$ and the over 6-month cohort ( $\mathrm{F}=6.27 \%, \mathrm{M}=5.10 \%)$.

Conclusion Further analysis persistently displays increased female mortality percentages within both cohorts of infants. This recurrence is greater due to a greater population thereby; the expansion of data has yielded stronger correlations. With the help of PICANET epidemiologist, we are increasing this population size further and focusing on admissions related to respiratory infections.

\section{G58(P) A NATIONAL AUDIT OF PARENTERAL NUTRITION PRACTISE IN UK NEONATAL INTENSIVE CARE UNITS: IS PRACTISE CONSISTENT WITH GUIDELINES?}

doi:10.1136/archdischild-2013-304107.070

${ }^{1} \mathrm{~A}$ Glynn, ${ }^{2} \mathrm{~S}$ Barr, ${ }^{3} \mathrm{~A}$ Lewis, ${ }^{3} \mathrm{DP}$ Tuthill. ${ }^{1}$ School of Medicine, Cardiff University, Cardiff, UK; ${ }^{2}$ Neonatal Unit, Cardiff \& Vale UHB, Cardiff, UK; ${ }^{3}$ Department of Paediatrics, Cardiff \& Vale UHB, Cardiff, UK

Background Parenteral nutrition (PN) is a lifesaving modality providing vital nutrients for neonates unable to tolerate enteral feeding. It has serious complications, including metabolic derangements, infection and line displacements which can be fatal. Positive outcomes can be maximised and complications minimised by appropriate biochemical monitoring, multidisciplinary involvement, adherence to evidence based clinical guidelines and careful venous line management.

Objective To audit current PN practises in all UK neonatal units against ESPGHAN European guidelines 2005 on protein and lipid introduction, American clinical guidelines for hyperglycaemia and 